\title{
Radiobiological evaluation of organs at risk for electronic high-dose-rate brachytherapy in uveal melanoma: a radiobiological modeling study
}

\author{
Timothy J. Waldron, MS!, Bryan G. Allen, MD, PhD', Edward Pennington, MS!, H. Culver Boldt, MD², Yusung Kim, PhD' \\ 'Departments of Radiation Oncology, Carver College of Medicine, The University of lowa, lowa City, lowa, USA, ${ }^{2}$ Departments of \\ Ophthalmology, Carver College of Medicine, The University of lowa, lowa City, lowa, USA
}

\begin{abstract}
Purpose: The objective of this study was to examine feasibility of single- or hypo-fraction of high-dose-rate (HDR) electronic brachytherapy (eBT) in uveal melanoma treatment.

Material and methods: Biologically effective doses (BED) of organs at risk (OARs) were compared to those of iodine-125-based eye plaque low-dose-rate brachytherapy ( ${ }^{125}$ I LDR-BT) with vitreous replacement (VR). Single- or hypo-fractionated equivalent physical doses (SFEDs or HFEDs) for tumor were calculated from tumor BED of ${ }^{125} \mathrm{I}$ LDR-BT using linear-quadratic (LQ) and universal survival curve (USC) models. BED OARs doses to retina opposite the implant, macula, optic disc, and lens were calculated and compared among SFED, HFED, and ${ }^{125}$ I LDR-BT. Electronic BT of $50 \mathrm{kVp}$ was considered assuming dose fall-off as clinically equivalent to ${ }^{125} \mathrm{I}$ LDR-BT. All OARs BEDs were analyzed with and without silicone oil VR.

Results: For a single-fraction incorporating VR, the median/interquartile range of LQ (USC)-based BED doses of the retina opposite the implant, macula, optic disc, and lens were 16\%/1.2\% (33\%/4\%), 35\%/19.5\% (64\%/17.7\%), $37 \% / 19 \%(75 \% / 17.8 \%)$, and $27 \% / 7.9 \%(68 \% / 23.2 \%)$ of those for ${ }^{125}$ I LDR-BT, respectively. SFED tumor values were 29.8/0.2 Gy and 51.7/0.5 Gy when using LQ and USC models, respectively, which could be delivered within 1 hour. SFED can be delivered within 1 hour using a high-dose-rate eBT. Even four-fraction delivery of HFED without VR resulted in higher OARs doses in the macula, optic disc, and lens $(135 \sim 159 \%)$ than when using ${ }^{125}$ I LDR-BT technique. A maximum $p$-value of 0.005 was observed for these distributions.

Conclusions: The simulation of single-fraction eBT, including vitreous replacement, resulted in significantly reduced OARs doses $(16 \sim 75 \%)$ of that achieved with ${ }^{125}$ I LDR-BT.

J Contemp Brachytherapy 2021; 13, 5: 563-574 DOI: https://doi.org/10.5114/jcb.2021.110349
\end{abstract}

Key words: electronic brachytherapy, episcleral plaque, uveal melanoma, biologically equivalent dose, vitreous replacement.

\section{Purpose}

Ocular melanoma, while rare, is the most common primary intraocular malignancy in adults and the second most common type of melanoma [1-3]. In the United States, ocular melanoma incidence is approximately six per million. It develops from melanocytes found in the choroid, iris, uvea, or ciliary body. Uveal melanomas account for over $80 \%$ of these malignancies [2,3]. Treatment of uveal melanomas includes enucleation or radiation therapy. Radiation therapy may be delivered either via brachytherapy (BT) [4-17], external beam radiation therapy (EBRT), using proton beams or stereotactic radiosurgery with Gamma knife (Elekta Instruments, Stockholm, Sweden). Outcomes [18] are reported in terms of surviv$\mathrm{al}$, metastases, recurrence, and visual acuity, but there are few EBRT results that include doses to OARs within the optical apparatus [19-21].

Iodine-125-based eye plaque low-dose-rate BT $\left({ }^{125} \mathrm{I}\right.$ LDR-BT) has proven to provide superior vision retention and equivalent tumor control over conventional enucleation for ocular melanomas [10, 22-24]. However, its delivery typically takes 5 days, with a dose-rate of $0.35-0.83 \mathrm{~Gy} / \mathrm{h}$. In this study, we explored the expected radiobiologically equivalent doses of organs at risk (OARs) when an equivalent tumor dose and dose distributions were simulated to be delivered in less than 5 fractions, currently possible due to the advent of highdose-rate (HDR) electronic brachytherapy (eBT), which has an equivalent dose fall-off due to the similar energy range (avg., 20-35 keV) as ${ }^{125}$ I LDR-BT (28 keV). Because of
Address for correspondence: Yusung Kim, PhD, DABR, Clinical Professor, Department of Radiation Oncology, Carver College of Medicine, The University of Iowa, 200 Hawkins Drive, 01607PFP-W, Iowa City, IA 52242, USA, phone: +1 319-384-9406, fax: +1 319-356-1530, 凶 e-mail: yusung-kim@uiowa.edu
Received: 29.10 .2020

Accepted: 23.08 .2021

Published: 29.10 .2021 
the nature of HDR-eBT delivery, radiobiologically equivalent OARs doses are expected to be higher than those of ${ }^{125}$ I LDR-BT when HDR-eBT is delivered in a single-fraction. However, no literature has reported estimated OARs doses in HDR-eBT delivered in a single- or hypo-fractionated fashion. Vitreous replacement (VR)/vitreous substitution, is a common treatment for retinal detachment [25]. Natural vitreous body is a gelatin-like fluid composed mainly $(\sim 98 \%)$ of water [25]. In VR, it is often replaced with synthetic biocompatible oil, such as silicone, which has a gelatin-like viscosity of 1,000 cSt. Clinical series utilizing VR as an adjuvant to episcleral plaque therapy have been reported by McCannel and others [26-28]. Radiobiologically equivalent HDR-eBT OARs doses over ${ }^{125}$ I LDR-BT have also not been reported when a silicone oil VR is performed just prior to irradiation. To our best knowledge, this is the first study assessing radiobiologically equivalent OARs sparing simulating HDR-eBT delivery through radiobiological modeling studies. No eBT applicators or collimators allowing equivalent ${ }^{125} \mathrm{I}$ LDRBT dose distributions have been studied. The conformality of ${ }^{125} \mathrm{I}$ LDR-BT is highly dependent on the choice of plaque size, specific loading or strength of individual ${ }^{125} \mathrm{I}$ source. To estimate comparative radiobiologically equivalent OARs doses from HDR-eBT, we did not change the plaque size, specific loading, or source strengths. Using clinical data of 284 patients, we noted distances between the ${ }^{125}$ I LDR-BT eye plaque and each of the OARs (retina, macula, optic disk, and lens). These structures are presented in the left panel of Figure 1A. The right panel of Figure 1B shows a representative cross section of an eye with a plaque in place and locations according to the collaborative ocular melanoma study (COMS) dose points used for dose calculations [29, 30]. These distances were applied to estimate radiobiologically-equivalent OARs doses for HDR-eBT based upon the findings of equivalent dose fall-off between ${ }^{125} \mathrm{I}$ and eBT. To normalize radiobiological effect of differences in fractionation schemes (i.e., continuous delivery of ${ }^{125}$ I LDR-BT versus single- or hypo-fractionated HDR-eBT delivery), radiobiologically equivalent OAR doses were computed using a widelyaccepted linear-quadratic (LQ) and universal surviv- al curve (USC) [31] models. This was a radiobiological modeling study to estimate the feasibility of HDR-eBT application as a form of intra-operative brachytherapy. To assess the feasibility of single-fraction HDR-eBT use in ocular melanoma treatment, corresponding OARs doses in biologically effective doses (BED) and equivalent dose in $2 \mathrm{~Gy}$ fraction $\left(\mathrm{EQD}_{2}\right)$ with and without VR were evaluated as the first step.

\section{Material and methods}

\section{Iodine-125 eye plaque brachytherapy patients}

A total of 284 patients treated for ocular melanoma with ${ }^{125}$ I LDR-BT episcleral eye plaques between February 22, 2006 and March 2, 2016 were retrospectively examined after an internal review board approval. All ${ }^{125} \mathrm{I}$ LDR-BT procedures were performed without VR, and current standard of care was used as a ground truth for this radiobiological modeling comparison study. Posterior tumors located sufficiently close to the optic nerve were treated with a 'notched' plaque, where the notch was an opening in the plaque surrounding the optic nerve. The prescription dose for all patients was $85 \pm 1.0 \mathrm{~Gy}$, delivered over approximately 120 hours in accordance with the COMS protocol [22]. Plaque loading with ${ }^{125}$ I sources was determined for each case with the tumor height and general shape details, as reported by an ophthalmologist. A radiation dose was prescribed to a point near the tumor apex. Tumor height or distance to the prescription point was measured from the inner scleral surface to the apex of tumor, with a $1 \mathrm{~mm}$ expansion in height. For the purpose of this study, doses were assumed to be similar to the surface and sclera $1 \mathrm{~mm}$ depth, based on apparent similarities of their depth dose percentages between $50 \mathrm{kVp}$ source and encapsulated ${ }^{125} \mathrm{I}$ source. The plaque diameter was chosen to provide a $2 \mathrm{~mm}$ gross tumor volume/planning target volume (GTV/PTV), like expansion at the base of the tumor. Chord distances to the tumor and critical structures in the eye were provided by the ophthalmologist based on physical and ultrasound examinations of the patient, and were reported relative to the optic disk and fovea. The prescription tumor height
A

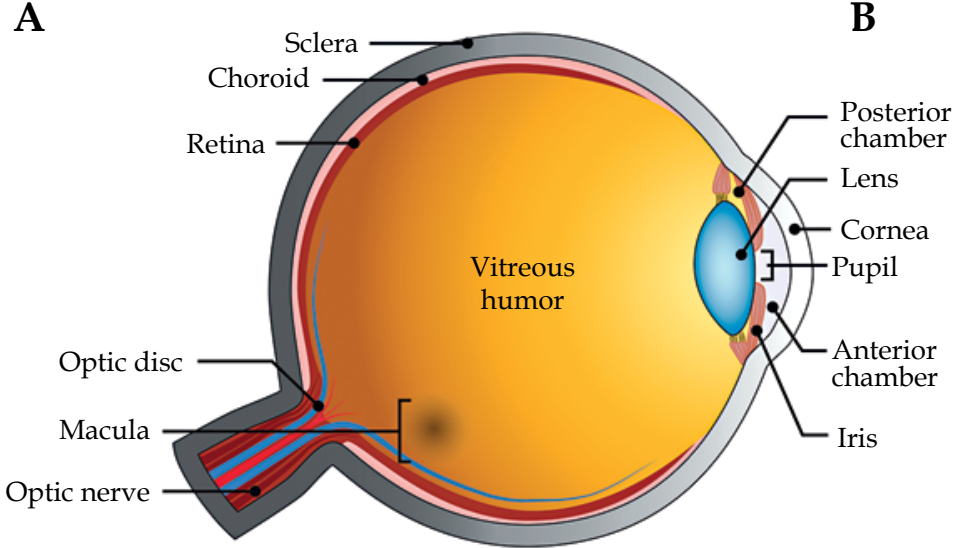

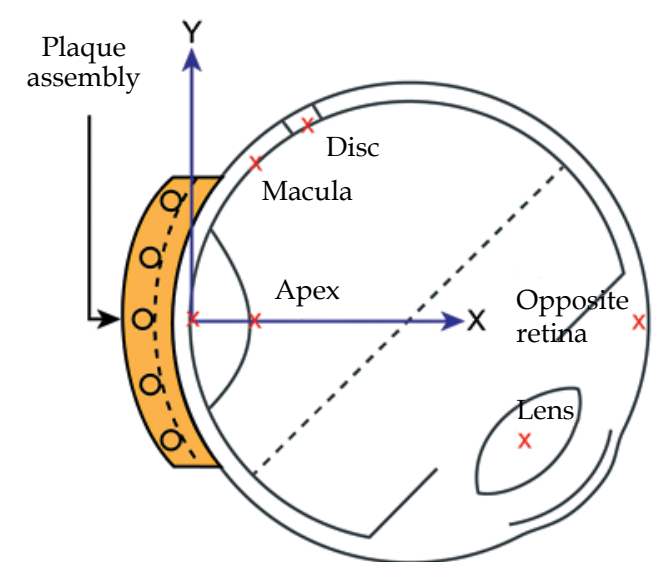

Fig. 1. General eye anatomy (A) and a schematic representation of a typical collaborative ocular melanoma study (COMS)

${ }^{125}$ I-based eye plaque brachytherapy (B) 
ranged from $2.2 \mathrm{~mm}$ to $11.3 \mathrm{~mm}$, with a median height of $4.3 \mathrm{~mm}$, of which, 205 were within $\pm 1.7 \mathrm{~mm}$ of this value. Distribution of prescription heights is shown in Table 1. Using the tumor edge to disk chord lengths and the tumor edge to fovea chord lengths with standard eye dimensions, relative distances between the target and interest points were determined. Seed locations for each plaque were known relative to the center of plaque. In relation to the anatomy of the eye, the plaque center was determined from tumor's spatial relationship to the fovea and optic disk. Model $6711^{125}$ I seeds (Amersham, GE Oncura, USA) were used up to June 2014. Subsequently, IsoAid model Advantage (IAI-125A, IsoAid, Port Richey, FL, USA) sources were used. Coordinates of the seeds relative to anatomical points of interest were entered into a treatment planning system (Pinnacle ${ }^{3}$ Treatment Planning, Philips Healthcare, Andover, MA, USA) to determine dose distribution. Dose calculations in this study were performed using a point source model according to the American Association of Medical Physics (AAPM) Task Group (TG) 43 formalism [32].

\section{BED dose calculations}

Prescription point doses of ${ }^{125}$ I LDR-BT were first converted into BED, based on a previous study [33]:

$$
\begin{aligned}
B E D_{125 I-B T} & =\left(\frac{R_{0}\left(1-\mathrm{e}^{-\lambda T}\right)}{\lambda}\right) \times\left\{1+\frac{2 R_{0} \lambda}{(\mu-\lambda)(\alpha \beta)\left(1-\mathrm{e}^{-\lambda T}\right)}\right\} \times \\
& \times\left[\frac{1}{2 \lambda}\left(1-\mathrm{e}^{-\lambda T}\right)-\frac{1}{(\mu+\lambda)}\left(1-\mathrm{e}^{-(\mu+\lambda) T}\right)\right] \quad \text { Eq. 1, }
\end{aligned}
$$

where $R_{0}$ is the initial dose rate, and $\lambda$ is the radionuclide-specific decay constant. The value of $\lambda$ is 0.0117 (1/day) and the value of $T$ represents implant time in hours. A mono-exponential recovery constant value of $\mu=0.46 \mathrm{~h}^{-1}$ was used for all OARs [33] and was calculated for $T_{1 / 2}=$ $1.5 \mathrm{~h}[34] . \alpha / \beta$ values of the opposite retina $=2.58$, macula $=2.58$, optic disc $=1.75$, and lens $=1.2$ were applied [33] The equivalent dose in 2 Gy fraction $\left(\mathrm{EQD}_{2}\right)$ of ${ }^{125} \mathrm{I}$ LDRBT was calculated (see Eq. 2).

$$
E Q D 2_{125 I L D R-B T}=\frac{B E D_{125 I L D R-B T}}{\left(1+\frac{2}{\alpha / \beta}\right)}
$$

Eq. 2.

The $\alpha / \beta$ of 11.5 was used for tumor BED and $\mathrm{EQD}_{2}$ calculations [33]. Then, single-fraction equivalent dose (SFED) or hypo-fraction equivalent dose (HFED) of HDReBT were estimated to deliver the same BED doses of ${ }^{125} \mathrm{I}$ LDR-BT (e.g., $B E D_{125 I \text { LDR-BT }}=B E D_{\text {HDR-eBT }}$ ). HDR-eBT $B E D$ values were recorded based on two different radiobiological models, including a conventional LQ model [35-37] shown in Eq. 3 and a USC model [31] presented in Eq. 3 or Eq. 4 , depending on dose per fraction $(d)$. USC model follows LQ model survival curve in Eq. 3 for low-dose range and multi-target model asymptote for high-dose range in Eq. 4. For continuity, there must be a single-transition dose $\left(D_{T}\right)$, at which point LQ model in Eq. 3 smoothly transitions to terminal asymptote of the multi-target model of Eq. 4. Therefore, when dose per fraction $(d)$ was lower than a single-transition dose $\left(D_{T}\right)$, the LQ and USC models were the same as in Eq. 3, while

\begin{tabular}{|c|c|c|}
\hline Characteristic & $n$ & $\%$ \\
\hline \multicolumn{3}{|l|}{ Patient age at implant } \\
\hline$\leq 50$ & 47 & 16.5 \\
\hline $50-69$ & 149 & 52.5 \\
\hline$\geq 70$ & 88 & 31.0 \\
\hline \multicolumn{3}{|l|}{ Tumor characteristics } \\
\hline \multicolumn{3}{|l|}{ Apical prescription height (mm) } \\
\hline $2.0-5.0$ & 175 & 61.6 \\
\hline $5.1-7.5$ & 83 & 29.2 \\
\hline 7.6-9.6 & 19 & 6.7 \\
\hline$>9.6$ & 7 & 2.5 \\
\hline Median height $(\mathrm{mm})$ & \multicolumn{2}{|c|}{4.3} \\
\hline \multicolumn{3}{|l|}{ Largest basal dimension (mm) } \\
\hline$\underline{5.0-8.0}$ & 40 & 14.1 \\
\hline $8.1-11.0$ & 86 & 30.3 \\
\hline $11.1-14.0$ & 101 & 35.6 \\
\hline $14.1-16.0$ & 57 & 20.1 \\
\hline Median basal dimension $(\mathrm{mm})$ & \multicolumn{2}{|c|}{12.0} \\
\hline
\end{tabular}

Table 1. Patient and tumor characteristics of this study

Closest tumor border to center of foveal avascular zone (FAZ) ( $\mathrm{mm})$

\begin{tabular}{lcc}
\hline 0.0 & 16 & 5.6 \\
\hline $0.1-2.0$ & 64 & 22.5 \\
\hline $2.1-5.0$ & 68 & 23.9 \\
\hline $5.1-7.5$ & 83 & 29.2 \\
\hline 7.5 & 26 & 9.2 \\
\hline
\end{tabular}

Median distance $(\mathrm{mm})$

Closest tumor border to edge of optic disk $(\mathrm{mm})$

\begin{tabular}{lll}
\hline $0.0-2.0$ & 74 & 26.1 \\
\hline $2.1-4.0$ & 54 & 19.0 \\
\hline $4.1-6.0$ & 55 & 19.4 \\
\hline $6.1-8.0$ & 38 & 13.4 \\
\hline$>8.0$ & 63 & 22.2 \\
\hline Median distance $(\mathrm{mm})$ & \multicolumn{2}{c}{4.90} \\
\hline Plaque characteristics & \multicolumn{2}{l}{}
\end{tabular}

Plaque characteristics

Standard plaque diameters $(\mathrm{mm})$

\begin{tabular}{lcc}
\hline 10.0 & 2 & 0.7 \\
\hline 12.0 & 7 & 2.5 \\
\hline 14.0 & 24 & 8.5 \\
\hline 16.0 & 69 & 24.3 \\
\hline 18.0 & 57 & 20.1 \\
\hline 20.0 & 47 & 16.5 \\
\hline 22.0 & 0 & 0.0
\end{tabular}

Notched/non-standard plaque diameters $(\mathrm{mm})$

\begin{tabular}{lcc}
\hline 10.0 & 0 & 0.0 \\
\hline 12.0 & 2 & 0.7 \\
\hline 14.0 & 5 & 1.8 \\
\hline 16.0 & 27 & 9.5 \\
\hline 18.0 & 19 & 6.7 \\
\hline 20.0 & 20 & 7.0 \\
\hline 22.0 & 5 & 1.8
\end{tabular}


the USC model followed Eq. 4 when the dose per fraction $(d)$ was larger than a single-transition dose $\left(D_{T}\right)$. The single-transition dose $\left(D_{T}\right)$ was estimated as 6.2 Gy [31] for non-small-cell lung cancer (NSCLC). Also, the LQ model was well validated, experimentally and theoretically, up to about 10 Gy per fraction, and would be satisfactory for usage up to about $18 \mathrm{~Gy}$ per fraction [38]. The value for $D_{T}$ has not been reported for uveal melanoma; therefore, the impact of $D_{T}$ values on the findings of this present study (i.e., OARs sparing of HDR-eBT) was examined by using $D_{T}=6.2,10$, and 18 Gy. However, these values did not affect the outcomes of this study significantly. The result of using $D_{T}$ value of 10 Gy was presented in this report, while the results of $D_{T}$ values of $6.2 \mathrm{~Gy}$ and $18 \mathrm{~Gy}$ were enclosed in the Appendix.

$$
B E D_{H D R-e B T}=N d\left(1+G \frac{d}{\alpha / \beta}\right)
$$

where $N$ and $d$ represent the number of hypo-fractions and dose per fraction. $G$ refers to a repair function that has a value of 1 for HDR single- or hypo-fractionated radiotherapies, such as EBRT or HDR-BT. When $N$ is 1 (single-fraction), $d$ is named SFED. Hypo-fractionated cases of $N=2,3$, and 4 were evaluated, in which $d$ was denoted as HFED.

$$
B E D_{H D R-e B T}=\frac{N\left(d-D_{q}\right)}{\alpha D_{0}}
$$

Where $d$ is still dose per fraction, $D_{0}$ and $D_{q}$ are the parameters that determine the final 'slopes' and $x$-intercept of the survival curve.

\section{OAR dose estimation of single- or hypo-fractionated electronic brachytherapy}

HDR-eBT $50 \mathrm{kVp}$ devices, such as Xoft Axxent (iCAD, Inc., Nashua, NH, USA) or IntraBeam (Carl Zeiss Meditec Inc., Oberkochen, Germany), present similar energy (avg., 20-35 keV) to that of ${ }^{125} \mathrm{I}$, and allow clinically similar high gradient isodose lines (i.e., radial functions) from a single-source, but with significantly higher dose rates of up to $60-114 \mathrm{~Gy} / \mathrm{h}$ at $20 \mathrm{~mm}$ [39]. In general, the ratio of physical doses to OARs relative to a physical tumor or prescription dose is determined largely by the implant geometry. For the purpose of this study, the equivalent dose distributions between HDR-eBT and ${ }^{125}$ I LDR-BT were simulated to be equivalent in order to assess the impact of higher eBT dose rates on OARs sparing. Therefore, for a given implant geometry (i.e., each case of ${ }^{125} \mathrm{I}$ LDR-BT), the physical dose ratios of each OAR to the tumor were assumed to be invariant with respect to fractionation scheme (single-fraction or hypo-fractionation) or delivery method (125I LDR-BT or HDR-eBT). By using the ratio of OAR to tumor point dose for each ${ }^{125}$ I LDR-BT case, a physical dose for each OAR point was computed from HDR-eBT SFED and HFED values of tumor point obtained from Eq. 3 or Eq. 4. Simulated HDR-eBT delivered $\mathrm{BED}$ and $\mathrm{EQD}_{2}$ values and then calculated using the following $\alpha / \beta$ values [33]: the opposite retina $=2.58$, the macula $=2.58$, the optic disc $=1.75$, and the lens $=1.2$.

\section{Attenuation by silicone oil}

The above-mentioned calculations were repeated for each case in a database including a transmission factor that would account for attenuation by the presence of VR liquid, being silicone oil, with a viscosity of 1,000 centistokes (cSt) [40, 41]. Attenuation of $50 \mathrm{kVp}$ eBT (IntraBeam; Carl Zeiss Meditec, Inc., Germany) by 1,000 cSt silicone oil relative to distilled water was measured for several thicknesses of oil under good geometry conditions, and a rational polynomial was fitted to the measured data. The eBT probe (IntraBeam; Carl Zeiss Meditec, Inc., Germany) was equipped with a rudimentary collimator (Figure 2). The first portion of the collimator was tubular, composed of copper and lead, and supported in a stand at $500 \mathrm{~mm}$ above the detector of thin-window parallel plate ion chamber (Exradin A11-TW; Standard Imaging, Inc., Middleton, WI, USA) (Figure 2). The first collimator had a larger inner diameter $(32 \mathrm{~mm})$ at its upper portion, with a smaller inner diameter $(22 \mathrm{~mm})$ at its final aperture. The
A

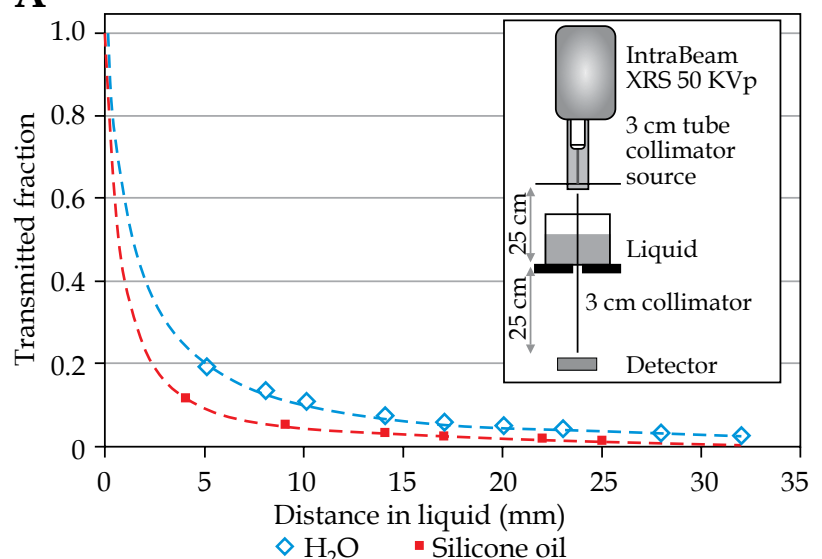

B

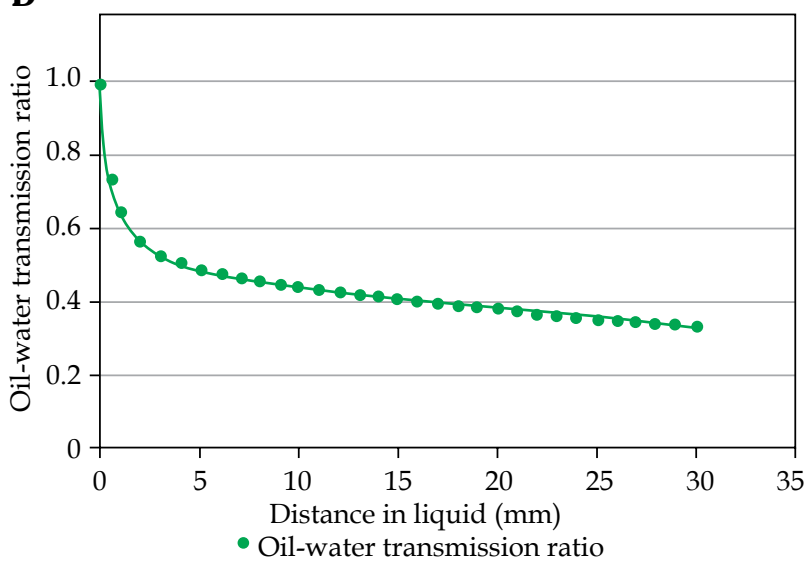

Fig. 2. Measurements of $50 \mathrm{kV}$ X-Rays transmission through water and silicone oil. The inset in the (A) shows the measurement setup. The (B) shows plots of the measured transmission through $1,000 \mathrm{cSt}$ silicone oil (squares) and distilled water at $\sim 22^{\circ} \mathrm{C}$ (diamonds). The right panel shows a plot of transmission ratio of silicone oil to distilled water with the fitted equation used for dose calculations 
upper portion of the first collimator was a brass/copper tube, with a $38 \mathrm{~mm}$ outer diameter and a $75 \mathrm{~mm}$ length. A tapered cone of $2 \mathrm{~mm}$ thick lead extended the length up to $185 \mathrm{~mm}$, where the lower portion of the copper tube was held as a final aperture. This placed the $22 \mathrm{~mm}$ inner diameter aperture at $20 \mathrm{~mm}$ distal to the probe tip. A circular collimator of $30 \mathrm{~mm}$ inner diameter was placed at $250 \mathrm{~mm}$ from the probe tip. A sheet of clear mylar of $0.16 \mathrm{~mm}$ thick was placed on top of the $30 \mathrm{~mm}$ collimator. A transparent plastic cylinder of $75 \mathrm{~mm}$ diameter was located on top of the mylar as a container of distilled water or silicone oil, and covered with clear plastic wrap of less than $0.005 \mathrm{~mm}$ thickness. Ionization current was measured at a series of depths from $4 \mathrm{~mm}$ to $28 \mathrm{~mm}$ for distilled water and 1,000 cSt silicone oil. Collected data were normalized to the measured value, with no liquid present, and each fitted to a rational polynomial expression. Attenuation ratios found for silicone oil to distilled water as a function of depth are presented in Figure 2. For each of the clinical cases examined in the present study, the vector distance from the implant origin to each of OAR dose points was used together with the fitted expression to compute a transmission factor for each point. Radiobiological calculations and analysis above were conducted as before, using the recomputed OAR doses.

\section{Statistical correlation analysis}

Wilcoxon signed-rank test was applied to evaluate skewness of distributions and degree of dependence between percentage of OARs doses calculated with radiobiological models and fractional physical OARs doses. Statistical analyses were performed using Excel (Microsoft Corporation, Redmond, WA, USA) and MATLAB (The MathWorks, Inc., Natick, MA, USA).

\section{Results}

The collected data exhibited significant skewness, thus were expressed as median and interquartile range (IQR). There were 146 treatments of the left eye, and 138 treatments of the right eye. The median (IQR) largest basal dimension within the 284 patients was recorded as 12.0 (4.0) $\mathrm{mm}$ (Table 1). The closest tumor border to the foveal avascular zone and the edge of the optic disk were observed as $4.5(6.0) \mathrm{mm}$ and $4.9(6.0) \mathrm{mm}$, respectively. The median plaque diameter was $18.0(4.0) \mathrm{mm}$. COMS plaque diameters used were from 10 to $22 \mathrm{~mm}$, and included both 'notched' or non-standard (78) and 'non- notched' or standard (206) plaques. Patients, tumors, and plaque characteristics are presented in Table 1 . The median total activity and implant times were $45.4 \pm 20.1 \mathrm{U}(40.1$ $\pm 15.8 \mathrm{mCi}$ ) and $120.1 \pm 13.7 \mathrm{~h}$, respectively. The doses at the prescription and OARs points in Gy of the $286^{125} \mathrm{I}$ LDR-BT patients are shown in Table 2. The median doserate at the prescription point was $0.73(0.03) \mathrm{Gy} / \mathrm{h}$. The median physical doses to OARs, including foveola/macula ('macula'), optic nerve/disk ('optic nerve'), center of the lens ('lens'), and retina opposite plaque ('retina') were 46.3 (61.5) Gy, 40.2 (46.8) Gy, 14.7 (11.0) Gy, and 6.8 (3.0) Gy, respectively. Anatomic points of interest ('retina', 'macula', 'disk', and 'lens') and dose calculations were defined according to COMS protocol for each plaque. Dose calculation technique used did not account for the effects of silastic insert or plaque 'lip' in keeping with clinical practice for most of the cases in this cohort.

The single-fraction physical dose (median, $30 \mathrm{~Gy}$, range, 28-32 Gy) and 52 Gy (range, 48-59 Gy) were observed when LQ and USC models were applied, and was radiobiologically equivalent to an average of $85 \mathrm{~Gy}$ (range, 84-90 Gy) of ${ }^{125}$ I LDR-BT from two different radiobiological models. It was feasible to deliver this dose within 1 hour with $50 \mathrm{kVp}$ eBT (IntraBeam; Carl Zeiss Meditec, Inc., Germany). The tumor SFED values via USC and LQ models were 51.7 (0.5) Gy and 29.8 (0.2) Gy, respectively, when ${ }^{125}$ I LDR-BT BED values of 107.3 (0.2) Gy were converted into radiobiologically equivalent doses given in 1 fraction. However, one of the major concerns of single- or hypo-fractionated HDR-eBT delivery was the expected dose to OARs.

Single-fraction delivery via HDR-eBT with VR resulted in radiobiologically equivalent OARs doses that were significantly less than doses for ${ }^{125}$ I LDR-BT (Figure 3). The USC-based BED and EQD 2 median doses to the retina, macula, optic disc, and lens were 33\% (4.3\%), 64\% (17.7\%), $75 \%(17.8 \%)$, and $16 \%$ (23.2\%) of those for ${ }^{125}$ I LDR-BT, respectively. The LQ-based BED and $\mathrm{EQD}_{2}$ median doses of the retina, macula, optic disc, and lens were $16 \%(1.2 \%)$, $35 \%(19.5 \%), 13 \%(19.1 \%)$, and $9 \%(7.8 \%)$ of those for ${ }^{125} \mathrm{I}$ LDR-BT, respectively. The median doses of ${ }^{125}$ I LDR-BT with VR, as expected, were found significantly lower than the current standard of care; ${ }^{125}$ I LDR-BT without VR presented 35\% (0.8\%), 34\% (3.5\%), 32\% (2.9\%), and 33\% (2.4\%) for the retina, macula, optic disc, and lens (Figure 3). All $p$-values for these distributions were less than 0.005 .

Regardless of the use of either USC or LQ models, a single-fraction of HDR-eBT without VR resulted in a sig-

Table 2. Tumor and organs at risk point doses of ${ }^{125}$ I-based eye plaque brachytherapy obtained from dataset of 284 cases

\begin{tabular}{lccccc} 
& Rx point & \multicolumn{3}{c}{ Organ at risk (OAR) point } \\
\cline { 2 - 7 } & Tumor & Retina & Macula & Disk & Lens \\
\hline Median physical dose $(\mathrm{Gy})$ & 84.9 & 6.8 & 46.3 & 40.2 & 14.7 \\
\hline IQR of physical dose $(\mathrm{Gy})$ & 0.03 & 3.0 & 61.5 & 46.8 & 11.0 \\
\hline Median biological effective dose $\left(\mathrm{Gy}_{\mathrm{BED}}\right.$ ) & 107.2 & 7.4 & 74.2 & 74.2 & 21.5 \\
\hline IQR of biological effective dose $\left(\mathrm{Gy} \mathrm{BED}_{\mathrm{BE}}\right)$ & 1.04 & 3.6 & 158.6 & 135.7 & 21.7
\end{tabular}

$R x$ - prescription, Gy - Gray, Gy ${ }_{B E D}$ - Gray biologically effective dose, OAR - organ at risk, IQR - interquartile range 
A

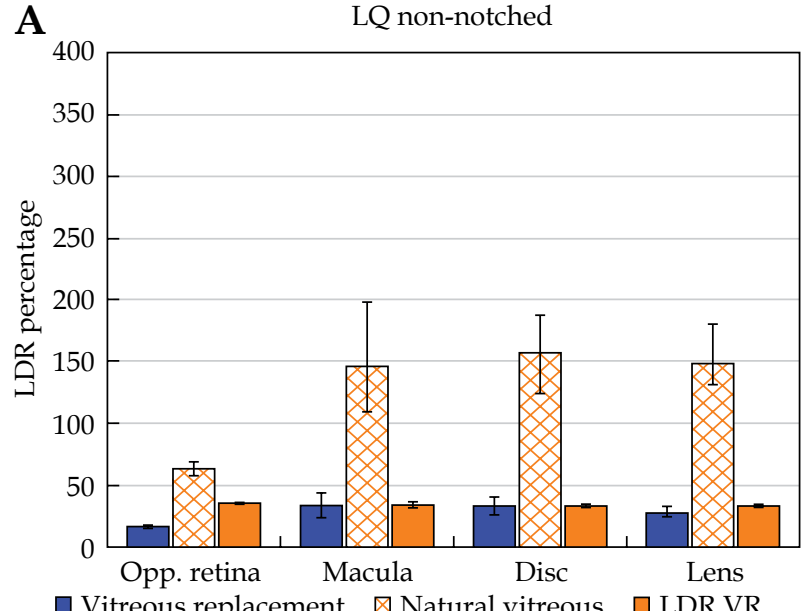

$\square$ Vitreous replacement $\quad$ Natural vitreous $\square$ LDR VR

C

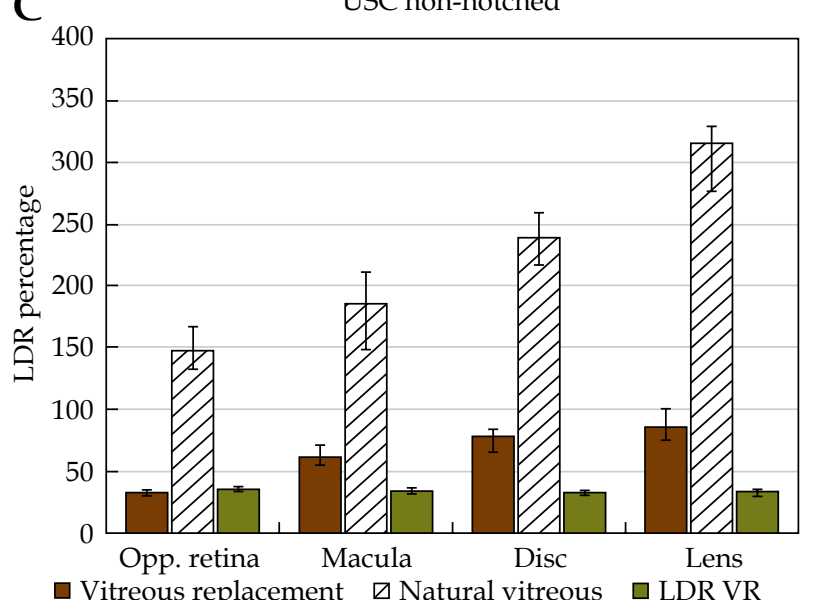

B

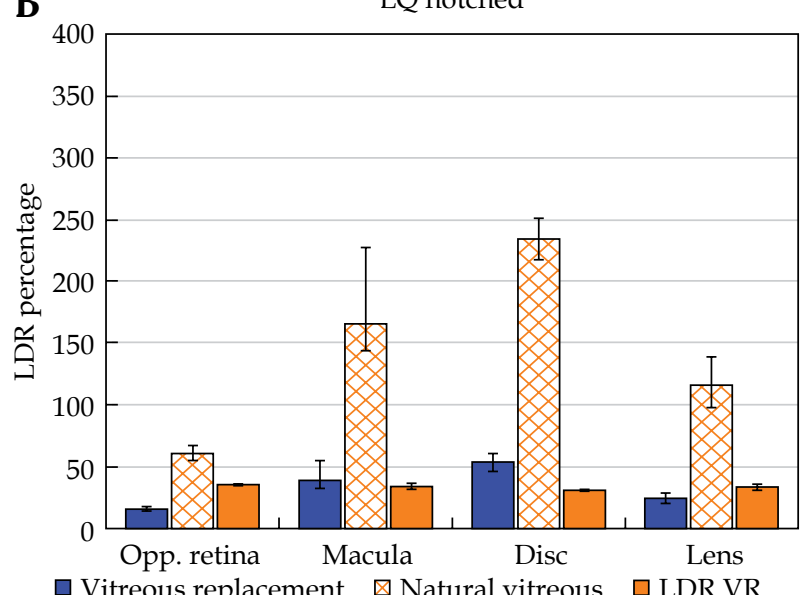

$\square$ Vitreous replacement $\quad$ Natural vitreous $\square$ LDR VR

D

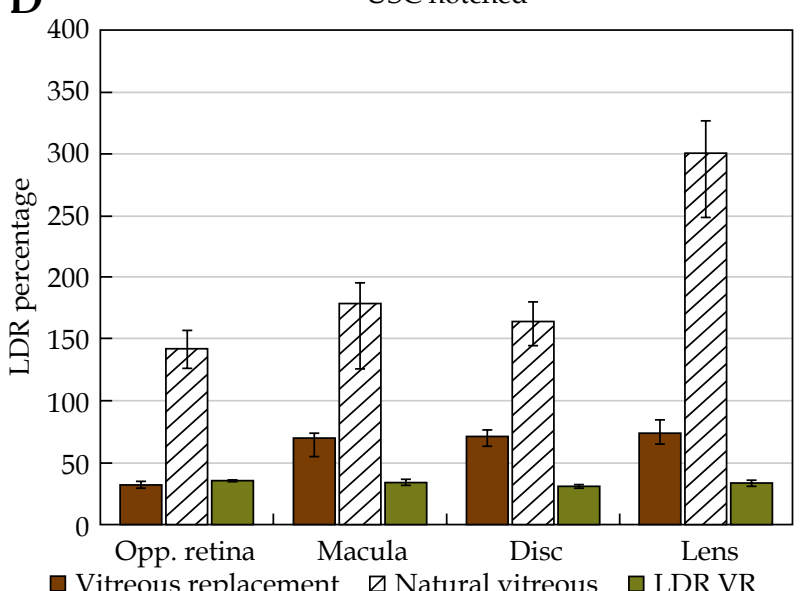

Fig. 3. Organs at risk (OARs) analysis of BEDs for treatments comparing simulations performed with vitreous replacement (VR) to those without (no-VR) for notched $(\mathbf{B}, \mathbf{D})$ and non-notched plaques $(\mathbf{A}, \mathbf{C})$. OARs data are presented for the L-Q model in the (A, B) and the USC model in the (C, D)

nificantly higher median OARs doses to the retina, macula, optic disc, and lens, being $62 \sim 147 \%, 156 \sim 183 \%, 175 \sim$ $222 \%$, and $130 \sim 313 \%$ of those for ${ }^{125}$ I LDR-BT, respectively. These differences in OARs doses between HDR-eBT and ${ }^{125} \mathrm{I}$ LDR-BT were significant $(p<0.005)$. The results for calculations of delivery involving 1-4 treatment fractions are summarized in Table 3. In delivery via 2-4 fractions for all the 284 cases without VR, the median OARs BEDs were from $69 \%(8.9 \%)$ to $77 \%(6.5 \%)$ of ${ }^{125}$ I LDR-BT plaque delivery for the retina, $147 \%$ (71.6\%) to $135 \%$ $(54.3 \%)$ for the macula, $162 \%(70.1 \%)$ to $146 \%$ (52.6\%) to the optic disk, and $125 \%(39 \%)$ to $119 \%(29.2 \%)$ for the lens using the LQ model. Using the USC model, the median OARs BEDs for 2-4 fractions were calculated from $106 \%(16.8 \%)$ to $89 \%(8.6 \%)$ of ${ }^{125}$ I LDR-BT plaque delivery for the retina, $162 \%(46.2 \%)$ to $137 \%$ (34.3\%) for the macula, $197 \%(49.0 \%)$ to $159 \%$ (35.1\%) for the disk, and from $207 \%$ $(55.9 \%)$ to $143 \%$ (35.3\%) for the lens. Notched plaque cases accounted for $27 \%$ of the total cases (78 of 284 cases) only, and therefore, could not be eligible due to the limited accommodation of an HDR-eBT applicator with a notched plaque. 206 of the 284 cases $(73 \%)$ studied were treated using nonnotched plaques, potentially HDR-eBT cases. For non- notched plaque treatments using VR, the median OARs BEDs computed for a single-fraction delivery using the LQ model were from $16.1 \%(1.2 \%)$ to $33.0 \%$ (20.1\%) relative to ${ }^{125}$ I LDR-BT plaque delivery BED, and those calculated using the USC model were from $33.2 \%(4.1 \%)$ to $86.1 \%(22.8 \%)$. Notched delivery cases utilizing VR calculated with the LQ model resulted in OARs BEDs from $15.9 \%(1.1 \%)$ to $53.9 \%$ (12.6\%) of ${ }^{125}$ I LDR-BT plaque BED. USC model calculations for notched plaques with VR showed OARs BED values from $32.5 \%$ (3.9\%) to $71.0 \%$ $(11.1 \%)$ of ${ }^{125}$ I LDR-BT plaque BED. Notched and nonnotched results for the LQ and USC models are compared in Figure 3.

Plaque sizes from $10 \mathrm{~mm}$ to $22 \mathrm{~mm}$ in a cumulative analysis of single-fraction HDR-eBT presented less than $75.0 \%$ of ${ }^{125} \mathrm{I}$ for all OARs BEDs, using either the LQ or USC models when VR was included. In contrast, all plaque sizes $(10 \sim 22 \mathrm{~mm})$ in HDR-eBT cumulative analysis showed more than $100 \%$ of ${ }^{125}$ I for at least one OAR BED, regardless of the use of LQ or USC model without VR. All HDR-eBT OARs BEDs had more than $146 \%$ of ${ }^{125} \mathrm{I}$ using the USC model, while the LQ model calculations resulted in more than $130 \%$ of ${ }^{125} \mathrm{I}$ for the macula, optic 
Table 3. BEDs as percentage of ${ }^{125}$ I LDR-BT eye plaque dose for treatments computed over various fractionations with and without vitreous replacement (VR) for organs at risk points studied in the current work. Median values from the 284 cases are shown

\begin{tabular}{|c|c|c|c|c|c|c|}
\hline Model & No. of fractions & Vitreous status & Retina (\%) & Macula (\%) & Disk (\%) & Lens (\%) \\
\hline \multirow[t]{8}{*}{ LQ } & \multirow[t]{2}{*}{1} & without VR & 61.9 & 156.4 & 175.2 & 130.1 \\
\hline & & with VR & 16.0 & 34.8 & 36.7 & 26.8 \\
\hline & \multirow[t]{2}{*}{2} & without VR & 68.7 & 146.7 & 162.0 & 124.8 \\
\hline & & with VR & 19.4 & 34.7 & 35.7 & 27.8 \\
\hline & \multirow[t]{2}{*}{3} & without VR & 73.3 & 140.0 & 153.1 & 121.2 \\
\hline & & with VR & 21.6 & 34.6 & 35.2 & 28.5 \\
\hline & \multirow[t]{2}{*}{4} & without VR & 76.8 & 134.7 & 145.8 & 118.5 \\
\hline & & with VR & 23.3 & 34.6 & 34.7 & 29.0 \\
\hline \multirow[t]{8}{*}{ USC } & \multirow[t]{2}{*}{1} & without VR & 146.7 & 182.8 & 221.5 & 313.5 \\
\hline & & with VR & 33.1 & 63.8 & 75.0 & 68.0 \\
\hline & \multirow[t]{2}{*}{2} & without VR & 106.3 & 162.3 & 197.3 & 207.1 \\
\hline & & with VR & 27.9 & 51.3 & 58.0 & 44.8 \\
\hline & \multirow[t]{2}{*}{3} & without VR & 94.2 & 146.9 & 172.8 & 165.7 \\
\hline & & with VR & 26.8 & 45.6 & 47.5 & 37.6 \\
\hline & \multirow[t]{2}{*}{4} & without VR & 89.2 & 137.3 & 159.2 & 143.1 \\
\hline & & with VR & 26.5 & 40.8 & 41.6 & 34.3 \\
\hline
\end{tabular}
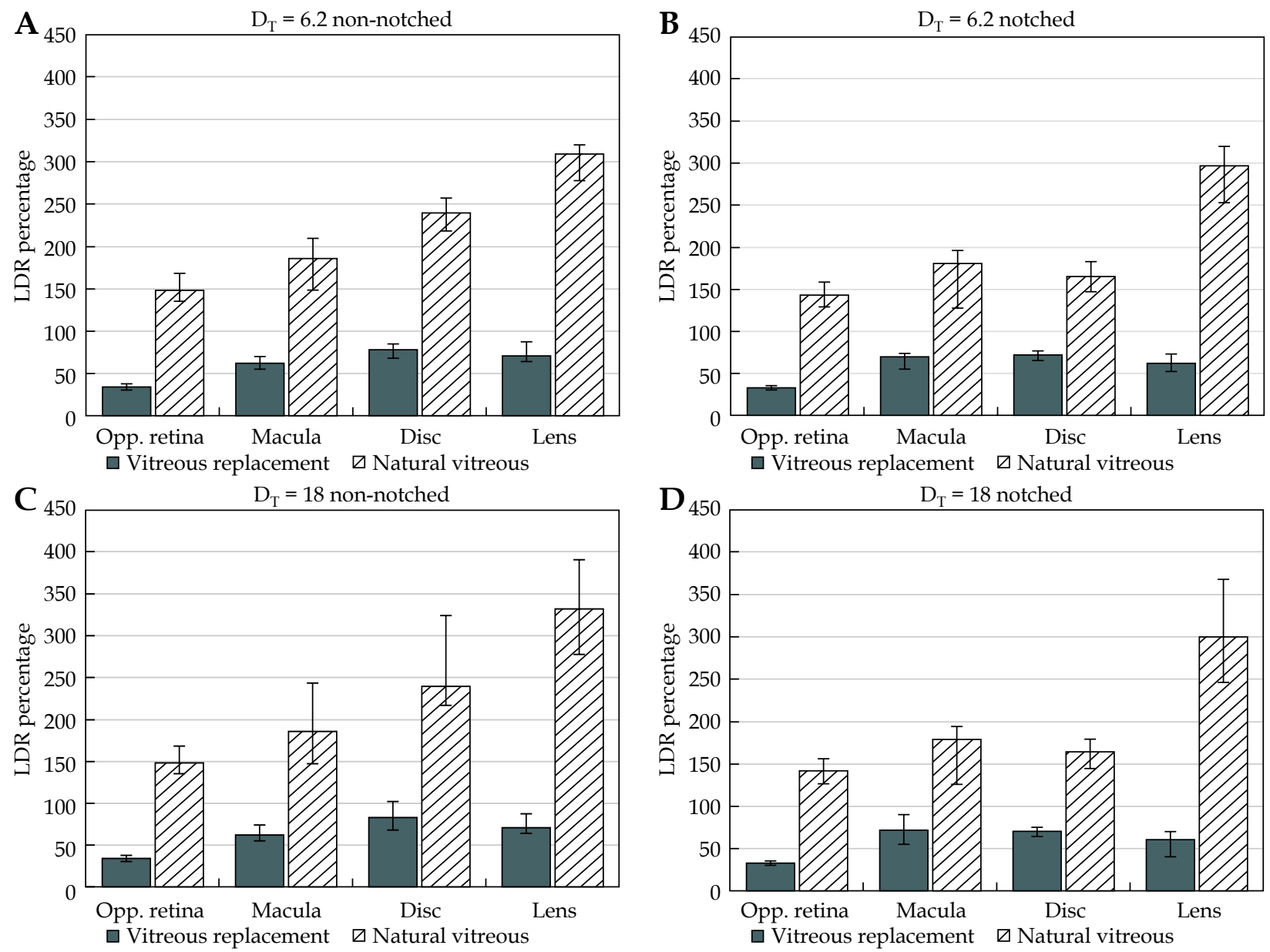

Fig. 4. A comparison of organs at risk (OARs) sparing of HDR-eBT when various single-transition doses are used. $D_{\mathrm{T}}=6.2 \mathrm{~Gy}$ in $\mathbf{A}$ and $\mathbf{B}$, and $\mathrm{D}_{\mathrm{T}}=18 \mathrm{~Gy}$ in $\mathbf{C}$ and $\mathbf{D}$. The USC model-calculated biologically effective doses (BEDs) as a percentage of ${ }^{125} \mathrm{I}$ LDR brachytherapy BEDs for cases without vitreous replacement (VR) (light diagonal lines) and with silicone oil VR (dark solid). Notched plaque data are plotted in $\mathbf{B}$ and $\mathbf{D}$, and non-notched in $\mathbf{A}$ and $\mathbf{C}$ 
disk, and lens when VR was not employed. The opposite retina BEDs were observed to be less than $100 \%$ of ${ }^{125} \mathrm{I}$ LDR-BT for all sizes without VR, but only with the use of LQ model. As plaque diameters increased from $10 \mathrm{~mm}$ to $22 \mathrm{~mm}$, the percentage of HDR-eBT over ${ }^{125}$ I LDR-BT $\mathrm{BED}$ for the retina ranged from $23 \%$ to $15 \%$ (24\% to $39 \%$ ) with the LQ model (USC model), and for the macula, it ranged from $23 \%$ to $44 \%$ ( $24 \%$ to $68 \%$ ) when VR was incorporated. The OARs sparing in SFED HDR-eBT with VR were observed to increase for the retina and the macula, as smaller plaque diameters (e.g., $10 \mathrm{~mm}$ diameter) were used for both the LQ and USC models. In contrast, no obvious trend with plaque diameter was observed for the optic disk and lens.

The sensitivity of the $D_{T}$ parameter on the USC-based $\mathrm{BED}$ and $\mathrm{EQD}_{2}$ calculations was evaluated using $D_{T}=6.2$ and $=18$, and calculated doses were compared with those using $D_{T}=10$. Regardless which $D_{T}$ value was used in the USC model, all OARs BEDs still presented less than 100\% of ${ }^{125} \mathrm{I}$ LDR-BT for a single-fraction HDR-eBT with VR. When three different values of the $D_{T}$ parameter were assessed, the percentage of HDR-eBT OARs BEDs over ${ }^{125} \mathrm{I}$ LDR-BT presented less than $0.3 \%$ and $1.1 \%$ differences for the macula and the optic disk, respectively, with no variation in the retina and the lens values. The impact of $D_{T}$ value on HDR-eBT HFED doses was also evaluated. As expected, HDR-eBT SFED with three different $D_{T}$ values produced the same value of 51.7 (0.5) Gy. The HFED doses with 2 fractions were still the same (26.7 (0.2) Gy) for all three $D_{T}$ values. For the 3 and 4 fraction cases, HFED doses with $D_{T}=18$ were smaller than those of $D_{T}=10$ and 6.2, which had the same HFED doses: $15.3(0.1) \mathrm{Gy}\left(D_{T}=18\right)$ vs. $18.4(0.2)$ Gy $\left(D_{T}=10\right.$ and 6.2) for 3 fractions, and 12.7 (0.1) Gy vs. 14.3 (0.1) Gy for 4 fractions.

\section{Discussion}

In the present study, we examined the feasibility of single- or hypo-fractions of HDR-eBT through a radiobiological model study. In anticipation of the development of HDR-eBT systems for uveal melanoma, one key question to the HDR-eBT approach is: 'Can HDR-eBT achieve the equivalent OARs sparing of the current standard-ofcare of a single-fraction of ${ }^{125}$ I LDR-BT?'. Moreover, 'If a single fraction does not work, how many fractions of HDR-eBT are needed to achieve the equivalent sparing?' and, 'If any fractionation of HDR-eBT does not work, then, how much sparing can be achieved using HDReBT with silicone oil VR?' To the best of our knowledge, this was the first radiobiological modeling study to assess OARs sparing of eBT-based HDR delivery for uveal melanoma compared to the current standard of ${ }^{125} \mathrm{I}$-based eye plaque brachytherapy with VR not performed. For the purpose of this study, higher strength ${ }^{125}$ I sources were not investigated, and a minimal eBT treatment system was proposed, in which the eBT source was applied similarly to a contact therapy. Here, a single $\mathrm{X}$-ray source is placed in a proximity, i.e., of $20 \mathrm{~mm}$ to the scleral surface, and a collimating applicator allows presentation of a 'beam' with a cross-sectional shape comparable to an episcleral plaque. This requires the eye to be positioned such that the collimator encompasses the tumor extent at the sclera. For very posterior tumors, including those necessitating a notched plaque, this positioning may only be achieved for a short period of time without unwanted tissue damage. Thus, treatment with such a system is likely impractical for tumors located in this region of the eye. Patients eligible for treatment with VR and nonnotched eye plaque candidates may derive some benefit from the proposed HDR-eBT approach. When an established intensity-modulated compensator approach is applied to HDR-eBT collimator design, then the HDR-eBT system is able to not only generate uniform dose distributions, but also potentially improve tumor coverage when the plan is based on magnetic resonance imaging (MRI) [6]. The eBT source in this study was simply a vehicle to explore the question of whether or not an HDR treatment can be considered with respect to radiobiological doses to OARs. The HDR source was not limited to eBT, but could be used with other radioactive sources, delivering HDR radiation with similar to ${ }^{125}$ I energy.

The proposed HDR-eBT delivers radiobiologically equivalent tumor doses of ${ }^{125}$ I LDR-BT through assessment of their estimated OARs BED values and comparison with those of ${ }^{125}$ I LDR-BT. A major benefit of the current ${ }^{125} \mathrm{I}$ LDR-BT technique is its low-dose-rate nature, which is selectively beneficial to normal tissue [19, 42], also confirmed by the findings in this study. Our results show that delivery of a single-fraction of high-dose-rate might be realized intra-operatively using eBT sources, and would result in unacceptably high biologically equivalent doses to OARs. This hypo-fractionated HDR approach using eBT technique was found to fail in achieving equivalent OARs doses in $\mathrm{BED}$ or $\mathrm{EQD}_{2}$ even with 4 fractions when VR was not applied. However, if a silicone oil VR was performed just prior to irradiation, the biologically equivalent OARs doses were significantly reduced in comparison to ${ }^{125} \mathrm{I}$ LDR-BT. Inclusion of VR may permit intra-operative treatment of accessible choroidal melanomas in a single-fraction HDR-eBT with lower OARs doses and a single surgical procedure, as opposed to a treatment duration of 5 days and 2 surgical visits.

The main purpose of this study was to examine the feasibility of a single-fraction HDR-eBT delivery of an equivalent tumor dose without increasing OARs doses through radiobiological modeling studies. The OARs doses of single-fraction HDR-eBT with and without VR were focused and compared to those of current ${ }^{125} \mathrm{I}$ LDR-BT. When the dosimetric results of a single HDReBT with VR are compared with ${ }^{125}$ I LDR-BT with VR, the LDR approach presents similar or even better OARs BEDs (Figure 3). However, the current COMS standard plaque design is such that it is difficult to limit the dose to non-target locations within the eye due to the fixed geometry. The current standard of ${ }^{125}$ I LDR-BT plaque consists of a gold backing with a silastic seed carrier insert available from 10-22 $\mathrm{mm}$ diameters in $2 \mathrm{~mm}$ increments [20]. The silastic insert has grooves to hold a fixed number of radioactive sources in place and to provide a relatively cylindrically symmetric dose distribution. In contrast to this, an HDR approach has the potential to use intensity- 
modulated brachytherapy (IMBT) techniques under investigation [43]. Callaghan et al. [43] systematically reviewed static and dynamic IMBT studies for cervical cancer, rectal cancer, breast cancer, and prostate cancer using eBT, ${ }^{192} \mathrm{Ir},{ }^{169} \mathrm{Yb}$, and ${ }^{153} \mathrm{Gd}$. In addition, current plaques must be sutured onto the outside of the sclera overlying the tumor and are left in place for 5 to 7 days until the prescribed dose is delivered. A second surgery is later performed to remove the plaque. This procedure is costly, since it requires two minor surgeries, possible hospitalization during the dose delivery period, and single-use brachytherapy sources. Also, a typical COMS ${ }^{125}$ I LDR-BT eye plaque results in a hand exposure of approximately 2-6 mSv to the surgeon per treatment [44]. A single-fraction HDR approach can omit the hospitalization as well as to minimize staff exposure.

In this radiobiological modeling study, the calculations of equivalent physical doses of single- or hypo-fractions were performed based on two (LQ and USC) radiobiological dose models. The LQ model that was first proposed and extensively studied by Fowler [45], is still the most widely accepted model to describe cell survival as a function of dose. However, one of its limitations is to describe cell survival for a large single-dose [31]. The USC model, which consists of two parts that depend on fraction size (dose per fraction) was also used in this study. When the fraction size is less than a certain threshold dose $\left(D_{T}\right)$, it yields an equivalent dose to the LQ model and therefore, reflects a multi-target effect when fraction size is larger than $\left(D_{T}\right)$ [8]. To estimate radiobiologically equivalent doses for stereotactic-body radiation therapy approaches, Park et al. validated the USC model in vitro by fitting it to a survival curve obtained for $\mathrm{H} 460$ non-small-cell lung cancer cell line [31]. The USC model may not be sufficiently validated through in vivo studies. The input parameters of neither the USC model nor the multi-targets model are available for uveal melanoma Due to these uncertainties, the sensitivity of the threshold dose $\left(D_{T}\right)$ was assessed for a wide range of its value $(D T=6.2 \sim 18 \mathrm{~Gy})$, while its standard value $(D T=10 \mathrm{~Gy})$ was employed in this study. The results of two different threshold doses (DT $=6.2$ Gy and $18 \mathrm{~Gy}$ ) are presented in the Appendix. Studies via in vitro and animal models should be followed to validate a hypothesis that is formulated by this preliminary radiobiological model-based dose calculation study. The radiobiological modeling considerations in ${ }^{125}$ I LDR-BT for uveal melanoma, such as its BED calculation and BED-based DVH, have been previously reported [33, 42]. A fundamental assumption, and therefore possible limitation of this study was that the relationships between the physical doses to the prescription point and the OARs points were invariant by virtue of geometry. This first-order approach may eventually be superseded by more complex models, which incorporate subtle biomaterial differences and other higher-order effects.

The geometry of individual tumors with respect to optical apparatus has a significant effect upon OARs doses. The macula, optic disk, and lens are very specific structures within the globe, whereas the retinal structure is distributed over a large portion of the interior surface. Therefore, a dose to the retina is less sensitive to tumor location than dose to the macula, optic disk, and lens. For beam-like irradiation, those structures located outside the projected beam are likely to receive lesser doses than those located on the beam path. Therefore, posterior structures (macula, optic disk) may receive higher doses from irradiation of anterior tumors than anterior structures (the lens), and conversely, irradiation of posterior tumors may involve greater dose to anterior structures and less to posterior structures. This variability in implant geometry may account for a large statistical uncertainties in our data for those categories with small sample sizes, such as the $10 \mathrm{~mm}$ plaque $(n=2), 12 \mathrm{~mm}$ plaque $(n=9)$, and $22 \mathrm{~mm}$ plaque $(n=5)$.

Geometry also strongly impacts the application of an HDR-eBT approach to ocular melanomas since the appositional geometry available with current HDR-eBT systems would require the eye to be rotated and immobilized sufficiently to permit beam access to the tumor via an anterior orbital fossa. In our clinical database of 284 patients, there were 78 cases $(27 \%)$ using notched plaques. The amount of rotation needed to make these accessible is likely impractical for HDR-eBT treatment. However, the remaining 206 cases (73\%) may have presented in a manner that permits consideration for an intra-operative treatment approach using eBT. It is expected that the findings of this study could encourage more research on single- or hypo-fractionated HDR-eBT applications for uveal melanoma. However, this study simulated radiobiologically equivalent OARs doses of HDR-eBT compared to ${ }^{125} \mathrm{I}$ LDR-BT, which is the current standard of care for uveal melanoma, typically performed without VR. For the purpose of this study, the current clinical standard of care of ${ }^{125}$ I LDR-BT without VR was applied as reference datasets to assess the feasibility of HDR-eBT in terms of OARs sparing. The actual application of HDR-eBT is highly dependent on the specific technique, including specific type of eBT applicator or collimation used. New applicators, collimators, and full system developments need to be explored, with their dosimetric properties validated through phantom studies.

To incrementally improve the uncertainty of eBT dose calculation, the use of formulas derived from TG-43 are an ideal way to calculate each HDR-eBT OAR dose. For the purpose of this radiobiological modeling study, the physical HDR-eBT OARs doses were estimated from the ratio of each OAR dose over the tumor dose in ${ }^{125}$ I LDRBT. The TG-43 source parameters of Xoft Axxent (iCAD, Inc., USA) are known [46]. In contrast to the Xoft Axxent device, the TG-43 source parameters of the IntraBeam device (Carl Zeiss Meditec Inc., Germany) have not been reported mainly due to the nature of its delivery (without treatment planning.) For instance, in IntraBeam-based intra-operative radiotherapy for accelerated breast irradiation, surface dose on each solid applicator with $20 \mathrm{~Gy}$ of prescription dose, is determined based on the distance from the electronic source. In the current study, the HDR eBT delivery was simulated using an IntraBeam device (Carl Zeiss Meditec Inc., Germany). When each eBT, OAR 
dose was calculated using TG-43 formalism, the absolute physical dose of each OAR in eBT could change. However, their average values in the investigated 284 cases were expected to be mainly driven by their geometrical distance from the tumor.

Once an integrated HDR-eBT system is completed, Monte Carlo dose calculation studies should be performed on the new system. Initially, dose distributions of a couple of representative ${ }^{125} \mathrm{I}$ eye plaque cases can be validated using the HDR-eBT system, and compared to those of ${ }^{125}$ I LDR-BT eye plaque doses using a Monte Carlo simulation. In order to deliver uniform doses to the target region on the proposed HDR-eBT collimator system, we can adopt the approach of 3D-printed solid compensators, which have been widely used in an external beam intensity-modulated radiotherapy.

In addition, the radiobiological effects of HDR-eBT on OARs of uveal melanoma should be validated through in vivo animal studies before any HDR-eBT clinical trial. Under an assumption of equivalent dose distribution, the retina, macula, optic disk, and lens were analyzed. If dose distributions differ, the sclera dose should also be validated via in vivo animal models. The findings of this study are expected to provide guidelines for a clinical study design to explore alternative dose-rate sources, and/or different fractionation schemes in order to reduce the current lengthy hospital stay.

\section{Conclusions}

Our results indicate that single-fraction equivalent doses using high-dose-rate eBT could be delivered within 60 minutes, and may significantly reduce OARs doses $(17 \% \sim 86 \%)$ relative to ${ }^{125}$ I LDR-BT when VR with 1,000 CSt silicone oil is simulated. Regardless of the use of either USC or LQ model, even four-fraction delivery of eBT without VR resulted in a significantly higher OARs doses $(p<0.005)$ to the macula, optic disc, and lens of those for ${ }^{125}$ I LDR-BT, respectively.

PACS Number: 87.19.xj, 87.50.cm, 87.50.ct, 87.53.Jw, 87.55.D-, 87.55.dk.

\section{Disclosure}

The authors report no conflict of interest.

\section{References}

1. Weersink RA, Patterson S, Ballantyne H et al. An improved treatment planning and quality assurance process for Collaborative Ocular Melanoma Study eye plaque brachytherapy. Brachytherapy 2019; 18: 658-667.

2. Krantz BA, Dave N, Komatsubara KM et al. Uveal melanoma: epidemiology, etiology, and treatment of primary disease. Clin Ophthalmol 2017; 11: 279-289.

3. Jovanovic P, Mihajlovic M, Djordjevic-Jocic J et al. Ocular melanoma: an overview of the current status. Int J Clin Exp Pathol 2013; 6: 1230-1244.

4. Morrison H, Larocque MP, Menon G et al. Delivered dose changes in COMS plaque-based ocular brachytherapy arising from vitrectomy with silicone oil replacement. Brachytherapy 2019; 18: 668-674.
5. Le BHA, Kim JW, Deng H et al. Outcomes of choroidal melanomas treated with eye physics plaques: A 25-year review. Brachytherapy 2018; 17: 981-989.

6. Zoberi JE, Garcia-Ramirez J, Hedrick S et al. MRI-based treatment planning and dose delivery verification for intraocular melanoma brachytherapy. Brachytherapy 2018; 17: 31-39.

7. Miguel D, de Frutos-Baraja JM, Lopez-Lara F et al. Radiobiological doses, tumor, and treatment features influence on outcomes after epiescleral brachytherapy. A 20-year retrospective analysis from a single-institution: part II. J Contemp Brachytherapy 2018; 10: 347-359.

8. Miguel D, de Frutos-Baraja JM, Lopez-Lara F et al. Radiobiological doses, tumor, and treatment features influence on local control, enucleation rates, and survival after epiescleral brachytherapy. A 20-year retrospective analysis from a single-institution: part I. J Contemp Brachytherapy 2018; 10:337-346.

9. Miguel D, de Frutos-Baraja JM, Lopez-Lara F et al. Visual outcome after posterior uveal melanoma episcleral brachytherapy including radiobiological doses. J Contemp Brachytherapy 2018; 10: 123-131.

10. Messer JA, Zuhour RJ, Haque W et al. Eye plaque brachytherapy versus enucleation for ocular melanoma: an analysis from the National Cancer Database. J Contemp Brachytherapy 2020; 12: 303-310.

11. Miguel D, de Frutos JM, Lopez-Lara F et al. Treatment planning considerations for (125)I eye plaque brachytherapy. J Contemp Brachytherapy 2019; 11: 280-284.

12. Rospond-Kubiak I, Wroblewska-Zierhoffer M, Twardosz-Pawlik $\mathrm{H}$ et al. Ruthenium brachytherapy for uveal melanoma - single institution experience. J Contemp Brachytherapy 2017; 9: 548-552.

13. Tagliaferri L, Pagliara MM, Boldrini L et al. INTERACTS (INTErventional Radiotherapy ACtive Teaching School) guidelines for quality assurance in choroidal melanoma interventional radiotherapy (brachytherapy) procedures. J Contemp Brachytherapy 2017; 9: 287-295.

14. Astrahan MA. The retina dose-area histogram: a metric for quantitatively comparing rival eye plaque treatment options. J Contemp Brachytherapy 2013; 5: 23-32.

15. Miguel D, Saornil MA, de Frutos JM et al. Regression of posterior uveal melanoma following iodine- 125 plaque radiotherapy based on pre-treatment tumor apical height. J Contemp Brachytherapy 2021; 13: 117-125.

16. Ces AP, Rodriguez MB, Sueiro JM et al. Intra-operative ocular ultrasonography of iodine-125 brachytherapy plaques in patients with uveal melanoma. J Contemp Brachytherapy 2021; 13: 126-134.

17. Karimi S, Arabi A, Siavashpour Z et al. Efficacy and complications of ruthenium-106 brachytherapy for uveal melanoma: a systematic review and meta-analysis. J Contemp Brachytherapy 2021; 13: 358-364.

18. Gorovets D, Gagne NL, Melhus CS. Dosimetric and radiobiologic comparison of (103)Pd COMS plaque brachytherapy and Gamma Knife radiosurgery for choroidal melanoma. Brachytherapy 2017; 16: 433-443.

19. American Brachytherapy Society - Ophthalmic Oncology Task Force. Electronic address pec, Committee AO. The American Brachytherapy Society consensus guidelines for plaque brachytherapy of uveal melanoma and retinoblastoma. Brachytherapy 2014; 13: 1-14.

20. Chiu-Tsao ST, Astrahan MA, Finger PT et al. Dosimetry of (125)I and (103)Pd COMS eye plaques for intraocular tumors: report of Task Group 129 by the AAPM and ABS. Med Phys 2012; 39: 6161-6184.

21. Finger PT. Radiation therapy for orbital tumors: concepts, current use, and ophthalmic radiation side effects. Surv Ophthalmol 2009; 54: 545-568. 
22. Design and methods of a clinical trial for a rare condition: the Collaborative Ocular Melanoma Study. COMS Report No. 3. Control Clin Trials 1993; 14: 362-391.

23. Jampol LM, Moy CS, Murray TG et al. The COMS randomized trial of iodine 125 brachytherapy for choroidal melanoma: IV. Local treatment failure and enucleation in the first 5 years after brachytherapy. COMS report no. 19. Ophthalmology 2002; 109: 2197-2206.

24. Hawkins BS, Collaborative Ocular Melanoma Study Group. The Collaborative Ocular Melanoma Study (COMS) randomized trial of pre-enucleation radiation of large choroidal melanoma: IV. Ten-year mortality findings and prognostic factors. COMS report number 24. Am J Ophthalmol 2004; 138: 936-951.

25. Kleinberg TT, Tzekov RT, Stein L et al. Vitreous substitutes: a comprehensive review. Surv Ophthalmol 2011; 56: 300-323.

26. McCannel TA, Kamrava M, Demanes J et al. 23-mm iodine-125 plaque for uveal melanoma: benefit of vitrectomy and silicone oil on visual acuity. Graefes Arch Clin Exp Ophthalmol 2016; 254: 2461-2467.

27. McCannel TA, McCannel CA. Iodine 125 brachytherapy with vitrectomy and silicone oil in the treatment of uveal melanoma: 1-to-1 matched case-control series. Int J Radiat Oncol Biol Phys 2014; 89: 347-352.

28. Ahuja Y, Kapoor KG, Thomson RM et al. The effects of intraocular silicone oil placement prior to iodine 125 brachytherapy for uveal melanoma: a clinical case series. Eye (Lond) 2012; 26: $1487-1489$

29. Scanderbeg DJ, Vasudev D, Rice RK et al. A modified COMS plaque for iris melanoma. J Contemp Brachytherapy 2011; 3 : 131-133.

30. Gagne NL, Cutright DR, Rivard MJ. Keeping an eye on the ring: COMS plaque loading optimization for improved dose conformity and homogeneity. J Contemp Brachytherapy 2012; 4: 165-175.

31. Park C, Papiez L, Zhang S et al. Universal survival curve and single fraction equivalent dose: useful tools in understanding potency of ablative radiotherapy. Int J Radiat Oncol Biol Phys 2008; 70: 847-852.

32. Nath R, Anderson LL, Luxton G et al. Dosimetry of interstitial brachytherapy sources: recommendations of the AAPM Radiation Therapy Committee Task Group No. 43. American Association of Physicists in Medicine. Med Phys 1995; 22: 209-234.

33. Gagne NL, Leonard KL, Rivard MJ. Radiobiology for eye plaque brachytherapy and evaluation of implant duration and radionuclide choice using an objective function. Med Phys 2012; 39: 3332-3342.

34. Dale RG, Jones B. The clinical radiobiology of brachytherapy. Br J Radiol 1998; 71: 465-483.

35. Fowler JF. Development of radiobiology for oncology a personal view. Phys Med Biol 2006; 51: R263-286.

36. Fowler JF, Dale RG. When is a "BED" not a "BED"?-When it is an EQD2: In regard to Buyyounouski et al. (Int J Radiat Oncol Biol Phys 2010;76:1297-1304). Int J Radiat Oncol Biol Phys 2010; 78: 640-641.

37. Fowler JF. 21 years of biologically effective dose. Br J Radiol 2010; 83: 554-568.

38. Brenner DJ. The linear-quadratic model is an appropriate methodology for determining isoeffective doses at large doses per fraction. Semin Radiat Oncol 2008; 18: 234-239.

39. Eaton DJ. Electronic brachytherapy - current status and future directions. Br J Radiol 2015; 88: 20150002.

40. Barca F, Caporossi T, Rizzo S. Silicone oil: different physical proprieties and clinical applications. Biomed Res Int 2014; 2014: 502143.

41. Oliver SC, Leu MY, DeMarco JJ et al. Attenuation of iodine 125 radiation with vitreous substitutes in the treatment of uveal melanoma. Arch Ophthalmol 2010; 128: 888-893.
42. Gagne NL, Leonard KL, Huber KE et al. BEDVH-A method for evaluating biologically effective dose volume histograms: application to eye plaque brachytherapy implants. Med Phys 2012; 39: 976-983.

43. Callaghan CM, Adams Q, Flynn RT et al. Systematic review of intensity-modulated brachytherapy (IMBT): static and dynamic techniques. Int I Radiat Oncol Biol Phys 2019; 105: 206-221.

44. Laube T, Fluhs D, Kessler C et al. Determination of surgeon's absorbed dose in iodine 125 and ruthenium 106 ophthalmic plaque surgery. Ophthalmology 2000; 107: 366-368; discussion 368-369.

45. Fowler JF. The linear-quadratic formula and progress in fractionated radiotherapy. Br J Radiol 1989; 62: 679-694.

46. Rivard MJ, Davis SD, DeWerd LA et al. Calculated and measured brachytherapy dosimetry parameters in water for the Xoft Axxent X-Ray Source: an electronic brachytherapy source. Med Phys 2006; 33: 4020-4032. 


\section{APPENDIX}

1. Radiobiological modeling of LQ and USC models.

SFED and HFED of HDR-eBT are simulated to be delivered in HDR mode; thus, repair function $(G)$ in Eq. 2 has the value of 1 . For the purpose of this radiobiological modeling study, the prescription point BED dose for HDR-eBT is established to have the same BED dose of ${ }^{125}$ I LDR-BT (i.e., $B E D_{H D R-e B T}=B E D_{125 I L D R-B T}$ ).

1A. SFED and HFED of HDR-eBT using LQ BED model.

Derivation of SFED and HFED are performed from the LQ model-based BED in Eq. 3.

$$
\begin{aligned}
& B E D_{125 I L D R-B T}=B E D_{H D R-e B T}=N \times d\left(1+\frac{d}{\alpha / \beta}\right) \text { Eq. A-1. } \\
& \mathrm{d}=\frac{-N+\sqrt{\left(N^{2}+4 \frac{N}{\alpha / \beta} B E D_{125 I L D R-B T}\right)}}{2\left(\frac{N}{\alpha / \beta}\right)} \text { Eq. A-2. }
\end{aligned}
$$

SFED: when a fraction number $(N)$ has value of 1 , SFED is calculated from BED of ${ }^{125}$ I LDR-BT in Eq. A-3.

$$
\mathrm{SFED}=\frac{-1+\sqrt{\left(1+4 \frac{1}{\alpha / \beta} B E D_{125 I L D R-B T}\right)}}{2\left(\frac{1}{\alpha / \beta}\right)} \text { Eq. A-3. }
$$

HFED: when a fraction number has value greater than 1, HFED is calculated from BED of ${ }^{125}$ I LDR-BT in Eq. A-4.

$$
\text { HFED }=\frac{-N+\sqrt{\left(N^{2}+4 \frac{N}{\alpha / \beta} B E D_{125 I L D R-B T}\right)}}{2\left(\frac{N}{\alpha / \beta}\right)} \text { Eq. A-4. }
$$

1B. SFED and HFED of HDR-eBT using USC BED model.

Fraction dose $(d) \leq$ a single-transition dose $\left(D_{T}\right)$ :SFED and HFED of HDR-eBT are calculated using Eq. A-3 and Eq. A-4, respectively.

Fraction dose $(d)>$ a single-transition dose $\left(D_{T}\right)$ : The USC model-based BED in Eq. 4 was derived from Eq. 5-B in [25], $\ln S=\frac{-d+D q}{D_{0}}$ where $N=1$. Thus, for the fractionated delivery, the USC model is given as Eq. A-5.

$\ln S=-N\left(\frac{d-D q}{D_{0}}\right)$ Eq. A-5.

$\ln S=-\alpha B E D, B E D_{H D R-e B T}$ is expressed in Eq. 2. From Eq. 2, the fraction dose $(d)$ is derived in Eq. A-6.

$d=\frac{N D_{q}+\alpha D_{0} B E D_{H D R-e B T}}{N}$ Eq. A-6.

a. SFED is calculated in Eq. A-7 when a fraction number $(N)$ has the value of 1.

$\mathrm{SFED}=D_{q}+\alpha D_{0} B E D_{125 I L D R-B T}$ Eq. A-7.

b. HFED is calculated in Eq. A-8 when a fraction number has the value of 2, 3, or 4 .

$\mathrm{HFED}=\frac{N D_{q}+\alpha D_{0} B E D_{H D R-e B T}}{N} \mathrm{Eq} . \mathrm{A}-8$.

2. Impacts of single-transition doses $\left(D_{T}=6.2 \mathrm{~Gy}\right.$ and $\left.18 \mathrm{~Gy}\right)$

The results were observed when two different single-transition doses $\left(D_{T}=6.2 \mathrm{~Gy}\right.$ and $\left.18 \mathrm{~Gy}\right)$ were used. Regardless of which single-transition doses $\left(D_{T}=6.2 \mathrm{~Gy}, 10 \mathrm{~Gy}\right.$, or $\left.18 \mathrm{~Gy}\right)$, single- or hypo-fractionated HDR-eBT was not able to achieve equivalent or better OARs sparing unless VR was placed. However, HDR-eBT with VR enables to spare OARs better than the current standard care of ${ }^{125} \mathrm{I}$ LDR-BT, despite the value of single-transition dose $\left(D_{T}\right)$. 